\title{
PHYLLODES TUMOUR: A CASE REPORT WITH REVIEW OF LITERATURE
}

Shaista Choudhary, Priyadarshini. M. M, Brahmacharimayum H. Sharma, Jayaprakash. H. T.

1. Associate Professor, Department of Pathology, Dr. B. R. Ambedkar Medical College.

2. Post Graduate, Department of Pathology, Dr. B. R. Ambedkar Medical College.

3. Post Graduate, Department of Pathology, Dr. B. R. Ambedkar Medical College.

4. Professor \& HOD, Department of Pathology, Dr. B. R. Ambedkar Medical College.

\section{CORRESPONDING AUTHOR}

Shaista Choudhary,

House No 60,

$10^{\text {th }}$ A Cross, West of Chord Road,

Bengaluru, Karnataka.

E-mail: drshaista5@rediffmail.com

Ph: 00919901474389

ABSTRACT: The term phyllodes tumour is recommended in World Health Organization (W.H.O) classification for the tumour previously called cystosarcoma phyllodes which means a large, fleshy tumour with a leaf like appearance on cut surface. [1]We report a case of phyllodes tumour in left breast of a 28 years old female patient. It was a huge tumour and resembled carcinoma clinically. A single left axillary lymph node was found to be enlarged. Fine needle aspiration was done and reported as phyllodes tumour which was later confirmed by histopathology. Lymph node cytology showed reactive changes. Though generally benign, phyllodes tumours have a tendency for recurrence. It can rarely become malignant too.

KEYWORDS: phyllodes tumour, benign, recurrence, lymph node

INTRODUCTION: Phyllodes tumour was earlier called as cystosarcoma phyllodes which was actually a misnomer. The currently preferred term is phyllodes tumour which is based on the tumour's leaf like appearance on cut surface. Johannes Miller in 1838 had named it as cystosarcoma phyllodes. ${ }^{[2]}$ The term 'sarcoma' in cystosarcoma caused a lot of confusion hence renaming it was most appropriate for the pathologists as well as the surgeons. Phyllodes tumour is most likely to reccur; some are locally aggressive and even metastasize. It occurs in older age group than fibroadenoma, median age being 45 years.[1] Phyllodes tumour constitutes less than one percent of total breast neoplasms and only 2-3\% of all fibroepithelial breast tumours. ${ }^{[3]}$ Phyllodes tumour usually present with a clinically palpable breast lump with wide variation in size. There can be bluish discolouration of skin with retraction of nipple and nodular as well as cystic feel on palpation. It can give a suspicion of malignancy at times. The etiopathogenesis of phyllodes tumour is not very clear. The treatment modality is surgery, depending on the size of tumour, type of tumour and if there is any lymph node metastasis.

CASE REPORT: A 28 year old female patient presented with a mass in left breast. Physical examination revealed a huge, firm mass with irregular margins and nodular areas. Entire mass measured $13 \times 10 \mathrm{cms}$. A lymph node was palpated in left axilla which was firm in consistency and measured $1 \mathrm{x} 1 \mathrm{cms}$ across. Mild nipple retraction was also noted.(fig-1) With these findings clinical index of suspicion was high. F.N.A.C of breast and axillary lymph node was done. 
Slides were stained with routine Leishman stain and Papanicolou stain. Smears from the breast mass showed biphasic pattern with admixture of stromal cells and ductal epithelial cells. The spindle shaped stromal cells were seen around the ductal epithelial cells.(fig-2,3) Smears from lymph node showed mixed cellularity, predominantly plasma cells, lymphocytes and immunoblasts.(fig-4)

Smears from breast mass were reported as Phyllodes tumour.

Smears from lymph node were reported as reactive lymphadenitis. The patient underwent mastectomy.Gross appearance was cauliflower like with nodular areas.(fig-5). Histopathology showed typical leaf like pattern with fibrous and spindle shaped stroma. Focal areas of stromal hypercellularity with benign glandular elements were also noted.(fig-6).Mitotic activity was $<5 / 10 \mathrm{hpf}$. Report was concluded with a diagnosis of benign phyllodes with reactive lymphadenitis of axillary lymph node.

DISCUSSION: Phyllodes tumours are rare fibroepithelial lesions that account for less than one percent $(1 \%)$ of all breast neoplasms. ${ }^{[4]}$ It has been called by 60 different names but presently the name Phyllodes tumour which is W.H.O approved is the most appropriate. It is a spectrum of fibroepithelial disorders rather than a single disease entity. At one end of spectrum is benign phyllodes tumour which is treated by local surgery and at the other end is malignant phyllodes which can reccur or metastasize, treatment being extensive surgery. Since the number of cases is small, there is lack of sufficient research data regarding the prognosis of disease. Etiopathogenesis of this tumour is quite unclear.

It has been suggested that in a proportion of fibroadenoma, a somatic mutation can result in a monoclonal proliferation, histologically indistinguishable from polyclonal elements, but with a propensity for local recurrence and progression to phyllodes tumour. Stromal proliferation is believed to be the result of growth factors produced by breast epithelium where endothelin-1 plays a major role.[4]Phyllodes tumour generally occurs at a older age compared to fibroadenoma i.e 38-48 years but cases in younger age has also been reported in literature. Phyllodes tumour has been classified into benign, borderline and malignant. The benign phyllodes tumour although do not metastasize but have the tendency to reccur locally. ${ }^{[5]}$ Although there are recommended criteria to guide the classification still the interpretation of individual histologic parameter and their integration into a final desision is still somewhat subjective.Many authors have used the degree of stromal hypercellularity and atypia, mitotic rate,stromal overgrowth and nature of microscopic margins as histologic features to stratify Phyllodes tumour into the respective grades. The presence of necrosis was also taken into account by Moffat et.al. and Reinfuss et.al, whereas Pietruszka and Barnes incorporated stromal overgrowth into their primary definition of Phyllodes tumour.[6]Tumours with morphology of fibroadenoma having acellular stroma without atypical features concentrated in the periductal areas are on the benign end of spectrum. Stromal component has fibroblastic appearance. Cytologically malignant phyllodes have marked nuclear atypia,numerous mitosis and loss of morphological relationship between glands and stroma.An important diagnostic criteria of malignancy is overgrowth of glands by sarcomatous stroma without epithelial elements. ${ }^{[2] M i t o t i c ~ a c t i v i t y ~ i s ~ f r e q u e n t l y ~ q u o t e d ~ a s ~ a ~ t h e ~ m o s t ~ i m p o r t a n t ~}$ feature in the differentiation of benign and malignant tumour. Many studies have shown the mitotic rate of more than 5/10HPF (high power field) indicates malignancy. However in the absence of any other worrying factor mitotic rate of 10/average $10 \mathrm{HPF}$ is most significant. Infiltrating margins, necrosis and hemorrhage also are crucial to diagnosis of malignancy. The 
term borderline tumours have been used on occasions for tumour wherein the microscopic distinction between benign and malignant characteristics is specially problematic. ${ }^{[1]}$ Nonetheless, accurate classification of Phyllodes continues to be challenging on many occasion. It could be due to the fact that Phyllodes tumour are morphologically heterogenous with different areas of the same tumour showing different appearences including areas morphologically resembling fibroadenoma.[7]Mammography of Phyllodes tumour typically shows lobulated benign appearing opacities. Clinical examination ,mammography, and even ultrasound does not allow a reliable diagnosis of Phyllodes tumour. ${ }^{[8]}$ Fine needle aspiration cytology may be more helpful provided there is adequate material in the smears.

In our case , the presentation was suspicious of malignancy (fig-1),since lymph node was also enlarged. Finally aspiration cytology and subsequently histopathology led to the diagnosis of benign phyllodes tumour.

The primary aim of treatment is complete surgical removal of tumour. Phyllodes tumour does not have a true capsule and therefore must not be enucleated. The extent of surgery depends on the preoperative diagnosis, tumour size its relation to breast size, age and patient preferences to a certain extent.[8,9] Larger the size of tumour, more the chances of total mastectomy. Certain cases show metastasis. Kesinger et.al. have reported metastasis $3-12 \%$ of cases. It was Lee and Pack in 1931, who reported the case of a patient with metastasis.[11]

CONCLUSION: Phyllodes tumour is a rare neoplasm of breast, majority being benign. careful search for the morphological features can provide accurate preoperative diagnosis(i.e. on F.N.A) which would help in planning surgery.

\section{REFERENCES:}

1. Darryl Carter, Stuart J. Schnitt,Rosemary R .Millis.The Breast.In:Sternberg's Diagnostic Pathology, th $^{\text {th }}$ edition;334-35.

2. Breast In:Rosai and Ackerman's Surgical Pathology.9th edition;1829-31.

3. Stamatakos M,Tsaknaki S,Kontzoglouk Gogas J,Kostakis A and Safioleas M.Phyllodes tumour of the breast: a rare neoplasm, though not that innocent.Int Seminars in surg oncol2009;6:6.

4. Parker S.J, Harrier S A.Phyllodes tumours. Postgraduate Medical jour2001; 77:428-36.

5. Aram F 0,Bafageer S S,BanafaN S.A huge phyllodes in 11 year old girl: A case report and review of literature.JBMS (jour of Behrain med society).2007;19:163-65.

6. TanPuay-Hoon,Jayabhaskar T,Chuah Khoon-Leong,Lee Hwei-Yee,Tan Yen,Hilmy Maryan et.al.Phyllodes tumours of the breast. Am Jour of clin pathol 2005; 123:529-40.

7. Dilip Giri: Recurrent challenges in the evaluation of fibroepithelial lesions. Arch Pathol Lab Med 2009; 133:713-21.

8. Stebbing J F, Nash A G.Diagnosis and management of phyllodes tumour of the breast: experience of 33 cases at a specialist centre. Ann R Coll Surg Engl 1995;77:181-84.

9. Imad A Hage,Aodah A,Kollur S M,Attallah A, Mohammed A.E and Al-Hussaini H.cytological clues in the distinction between phyllodes and fibroadenoma.Cancer Cytopathology 2010;118:33-40.

10. Badhe Bhawana A ,Iyengar Krishnan R,Alva Nandakishore.Astudy of fibroepithelial tumours of breast. Indian jour Cancer 2002; 39:91-96. 
11. Yamada Isao, Jino Yuichi and Yokoe Takao.Phyllodes tumours of the breast: A clinico pathological study of 118 cases. Jpn J of Surg.1997; 27:1137-43.

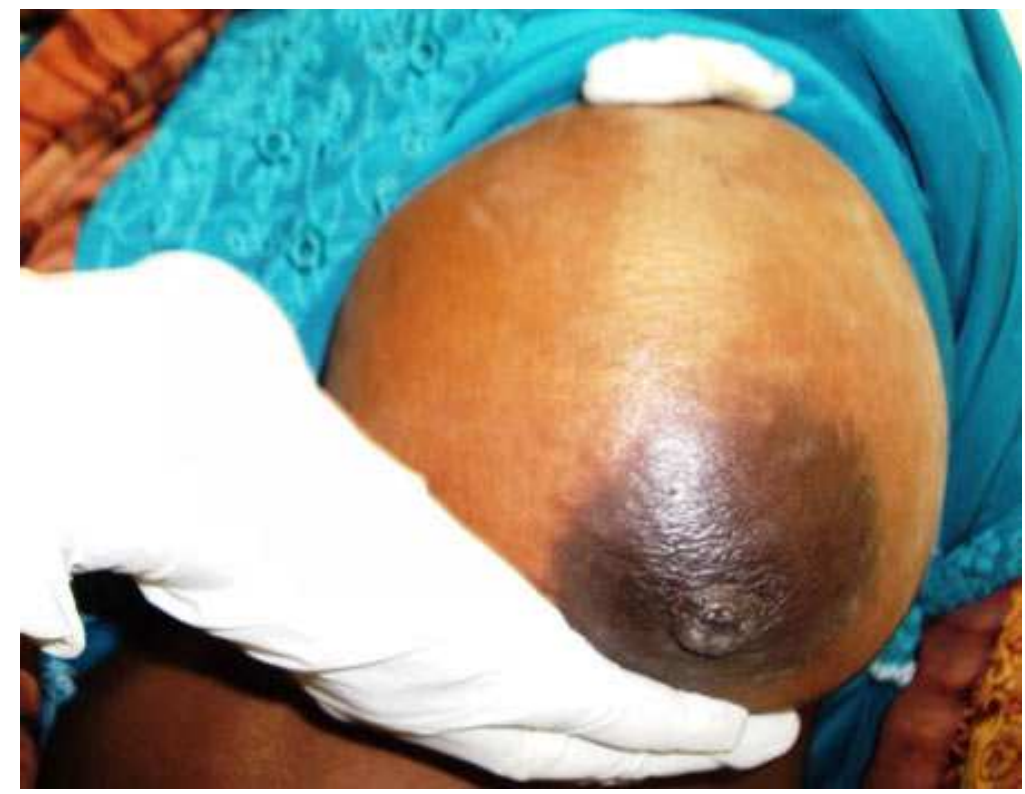

Figure 1- Enlarged breast on physical examination

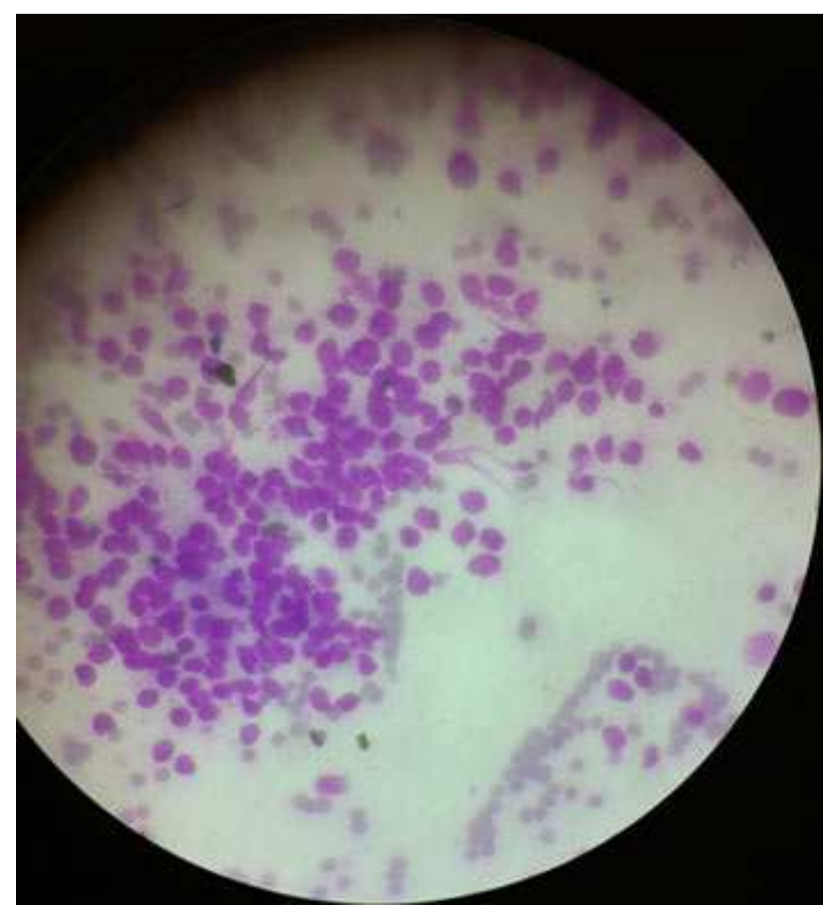

Figure 2- FNAC of the breast mass showing stromal cells and ductal epithelial cells. 


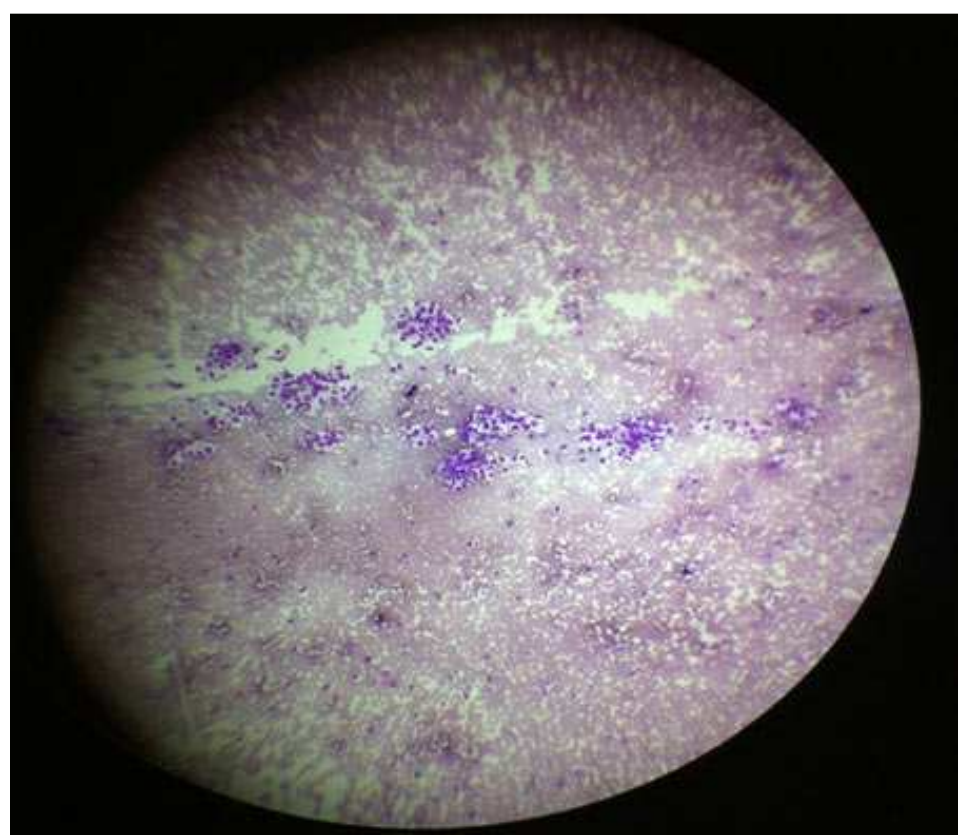

Figure 3- FNAC shows moderately cellular smears with spindle cells and ductal epithelial cells.

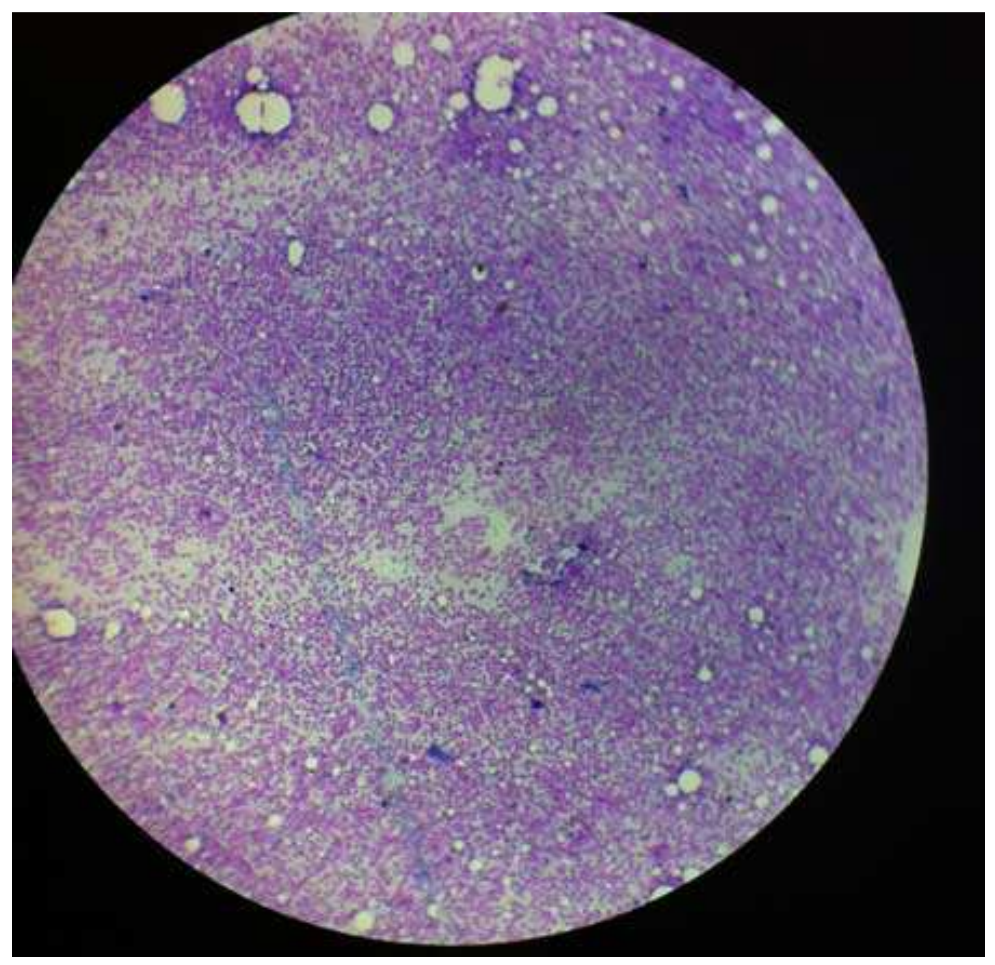

Figure 4- FNAC of lymph node showing features of reactive lymph node.(low power view) 


\section{CASE REPORT}

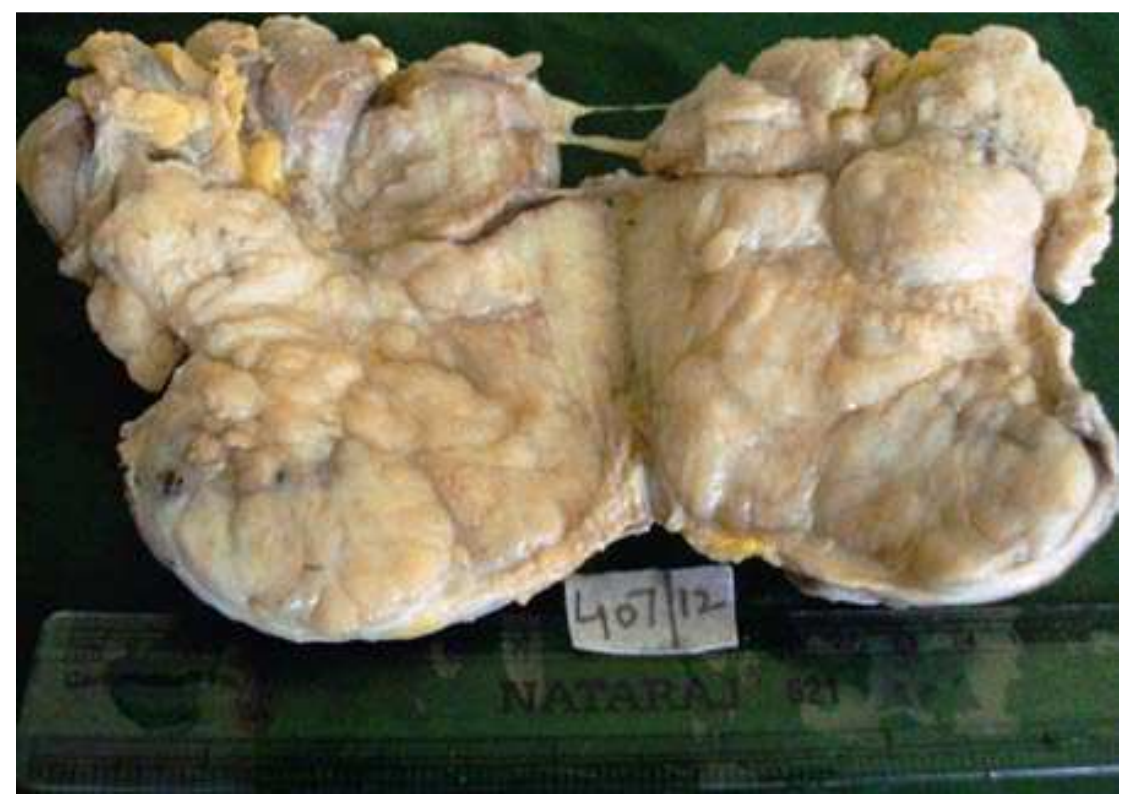

Figure 5- Gross morphology of breast mass showing nodules with cauliflower like appearance.

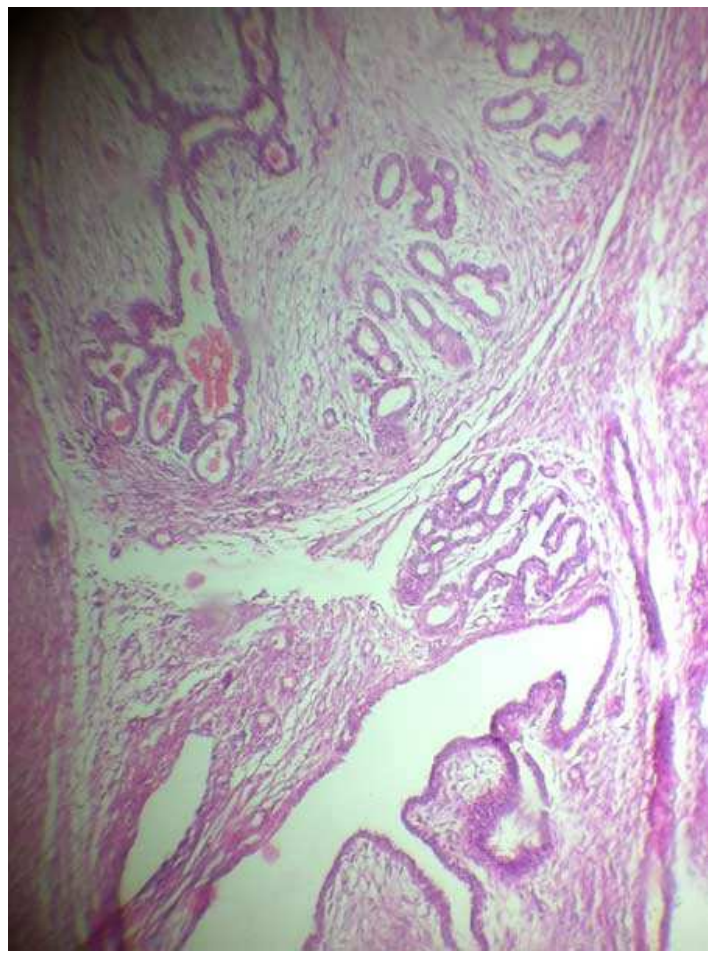

Figure 6- Histopathology showing biphasic pattern and typical leaf- like appearance. 